\title{
Percepciones de los docentes universitarios para la construcción de una cultura de paz
}

\author{
María Elsa Unriza Puin a \\ Isabel Hernández Arteaga ${ }^{b}$ \\ Fredys Aberto Simanca ${ }^{c}$
}

\section{Resumen}

Este artículo muestra los resultados de una investigación que busca determinar las percepciones que subyacen en la comunidad de docentes universitarios acerca de la construcción de una cultura de paz, en un proceso de transformación de una cultura de la violencia y con la responsabilidad de contribuir en la transmisión de elementos que genere cambio social. La investigación fue trabajada desde el paradigma cualitativo de tipo interpretativo y mediante el análisis de los resultados obtenidos por la triangulación de una encuesta no estructurada aplicada a los docentes, quienes participaron libre y voluntariamente. Se cuestionó acerca de los episodios que limitan o restringen la construcción de cultura de paz; igualmente, se buscó determinar si se considera que los colombianos confían en los acuerdos; y finalmente si la paz es experiencia de vida y forma parte de la cotidianidad. Los profesores, en su mayoría, coincidieron en manifestar que los colombianos no confiaban en el proceso de paz, porque este no era muy claro y tenía muchos enemigos; además, dijeron que los actores principales del conflicto actuaban a espaldas de la democracia y, peor aún, que le mintieron al pueblo. Se consideró la edad como la variable organizadora teniendo en cuenta varios elementos. Los periodos de violencia vividos por cada una de las edades fueron diferentes; el lenguaje utilizado por los docentes es distinto según el rango de edad. Las didácticas y pedagogías difieren entre una edad y otra; la construcción histórica de vida tiene diversas miradas según la experiencia y los años vividos.

Palabras clave: Percepciones. Cultura de Paz. Cambio Social. Paz. Docencia.

\footnotetext{
a Universidad Cooperativa de Colombia, Bogotá, Colombia.

b Universidad Cooperativa de Colombia, Bogotá, Colombia.

c Universidad Cooperativa de Colombia, Bogotá, Colombia. 


\section{Introducción}

En Colombia, la búsqueda de la paz, entendida no solo como ausencia de conflicto armado sino principalmente como el proceso de progreso, justicia y respeto mutuo (UNESCO, 1994), requiere necesariamente de la implementación de una cultura de paz.

Es necesario determinar una ruta que facilite e incluya los factores de dicha implementación. Se presentan varias alternativas, sin incluir las salidas violentas; se propone una cultura del manejo pacífico de conflictos "una perspectiva basada en una cultura del diálogo comunicacional, el entendimiento, los acuerdos y la actuación de valores" (ROJAS VERA, 2006).

En síntesis, se requiere de una cultura de paz que signifique ante todo un esfuerzo generalizado para modificar mentalidades y actitudes con ánimo de promover la paz (UNESCO, 1998). Significa transformar los conflictos y restaurar la paz y la confianza que se destruyen con la guerra.

Para la Organización de las Naciones Unidas para la Educación, la Ciencia y la Cultura (Unesco), la Educación es la punta de lanza de las actividades de promoción de una cultura de paz cuyo propósito trasciende los límites de los conflictos armados para hacerse extensivo también a las escuelas y los lugares de trabajo del mundo entero, los parlamentos, las salas de prensa, las familias y los lugares de recreo.

Las instituciones académicas tienen la responsabilidad de contribuir con el proceso de transformación de una cultura de la violencia a una cultura de paz. Los profesores, docentes, orientadores, son los encargados de la transmisión de elementos para la construcción de esta nueva sociedad.

(BERSTEIN, 1990 apud BOQUE TORREMORELL et al., 2014) consideran que la clave del discurso pedagógico se da, no por lo transmitido, sino por la transmisión, es decir por la forma como el docente emite la información, que es clave para la transformación cultural. Se requiere que toda comunidad oriente procesos hacia una cultura de paz y hacia el reconocimiento de las características individuales y sociales de sus integrantes, de las particularidades que les identifican y les permiten llegar a acuerdos y convivencia.

Solo se pueden establecer acuerdos o actuar según valores, cuando se conocen, reconocen y aceptan los elementos identitarios y culturales de todos los miembros 
de la comunidad en conflicto. Cada individuo le da sentido a su realidad según Bruner (1990) y Vygotsky (1978), a través del "pensamiento narrativo".

Andrews, (2007), Haste y Abrahams (2008) y Wertsch (1997) (apud PADILLA; BERMUDEZ, 2016) afirman que las personas adoptan narrativas disponibles en sus contextos socioculturales, como referencia para construir su comprensión de cuestiones sociales y su acción ante ellas; estas narrativas se convierten en teorías sociales que definen lo que se da por sentado, lo que se cuestiona, lo que se considera "natural" o "normal" y lo "problemático". Las narrativas ayudan a establecer coherencia entre identidades, experiencias individuales y colectivas. Los procesos narrativos en la Educación para la paz se concentran en explicar cómo las narrativas sociales afectan la explicación de los individuos sobre el conflicto, construyen representaciones sobre "el otro" a partir de interpretar sus acciones, y justificar la relación con sus "oponentes” (PADILLA; BERMÚDEZ, 2016, p. 233)

En este contexto, se busca entonces establecer: ¿Cuáles son las percepciones que subyacen en la comunidad académica universitaria, respecto de una cultura de paz? Concretamente se enfatiza en el cuerpo docente como sujeto transmisor, generador y motivador de imaginarios, y de la construcción de relatos, conceptos y acciones.

\section{Acercamiento teórico}

\subsection{La percepción}

La ruta que conduce a una cultura de paz como proceso naturalizado en una sociedad tiene elementos que facilitan los acuerdos, las creencias, valores y actitudes. Dentro de este ámbito se ubica la percepción que (VARGAS MELGAREJO, 1994) define como el proceso cognitivo de la conciencia mediante el reconocimiento, interpretación y significación para la elaboración de juicios en torno a las sensaciones obtenidas del ambiente físico y social, complementada con procesos como el aprendizaje, la menoría y la simbolización.

Esta definición, considerada como la de mayor validez y acercamiento a la construcción de pensamiento crítico hacia una cultura de paz, ha tenido procesos de complementación y diversificación que le dan un mayor alcance y nuevas propuestas y alternativas.

El reconocimiento que se hace de la percepción implica considerarla como un casi sinónimo de representación social, gestora de imaginarios, integrante de valores, generadora de conocimiento y determinante de inferencias, estructuras, primordiales para la construcción de ideologías y cultura. 
La responsabilidad es mayor cuando se trata de una comunidad académica, puesto que no se refiere solamente a una comunidad, sino que además es la encargada de la producción de conocimiento, de transmitir los valores culturales y de construir los procesos que conducen a la sociedad a un mejor vivir.

Igualmente, los procesos educativos, la escuela, las universidades, la academia en general, se presentan como estratégicos en la invención de la tradición, entendida, "como la creación de un relato que aglutina sentimientos, concepciones y símbolos que, a fuerza de repetición tienden a naturalizarse y reproducirse" (HOBSBAWM; RANGER, 1983 apud ARIAS GÓMEZ, 2016).

Es por lo que (BERSTEIN, 1990 apud BOQUE TORREMORELL et al., 2014) consideran que la clave de la transformación cultural es la transmisión del discurso pedagógico, más que el mismo contenido, es decir, la forma como dicho contenido es presentado. Esta afirmación es reforzada por Arias Gómez (2016) cuando cita a Freire (2001):

Por eso, para mí, uno de los contenidos esenciales de cualquier programa educativo, de sintaxis, de biología, de física, de matemáticas, de ciencias sociales, es lo que posibilita la discusión de la naturaleza cambiante tanto de la realidad natural como de la histórica y ve a los hombres y a las mujeres como seres que no solo son capaces de adaptarse al mundo, sino sobre todo, de cambiarlo, seres curiosos, activos, hablantes, creadores (p. 263).

En este sentido, identificar las percepciones que una comunidad tiene para la construcción de una cultura de paz implica valorar las creencias sobre las que están cimentadas sus acciones, reconocer los imaginarios que construyen sus pensamientos, comprender la simbología de sus representaciones sociales y entender las lógicas de sus actuaciones.

Este ejercicio permite un acercamiento que incluye una mayor valoración y respeto por el otro, por su ser autónomo individual, igualmente una construcción del colectivo en el que puedan incluirse, a través de consenso, la mayor cantidad de variables registradas. Las percepciones son esquemas de interpretación, valoración y clasificación del conocimiento del mundo, e incluyen las actitudes, los valores sociales y las creencias (VARGAS MELGAREJO, 1994). En ese mismo sentido, pero bajo otra estructura, acogiendo nuevos elementos (BOURDIEU, 2007) entiende las percepciones del mundo y las prácticas desde la noción de habitus, como: 
La capacidad infinita de engendrar en total libertad/controlada productos -pensamientos, percepciones, expresiones, acciones- que tienen siempre como límite las condiciones de su producción, histórica y socialmente situadas; la libertad condicionada y condicional que asegura está tan alejada de una creación de imprevisible novedad como de una simple reproducción mecánica de los condicionamientos iniciales (p. 90).

El habitus, desde las estructuras objetivas, genera sistemas de prácticas y representaciones, dando a la conducta esquemas básicos de percepción y de acción, modos de experimentar y clasificar lo real. El habitus es determinante en la forma de percepción. La idea del habitus de Bourdieu (1988) permite problematizar las relaciones sociales, desde el poder como el elemento que constituye lo social, y que a su vez estructura las percepciones y el conocimiento que los sujetos tiene del mundo. La percepciones y acciones del mundo se marcan desde la dotación de determinados habitus que el sujeto o actor social tengan para sí.

Bajo esos lineamientos, Kant considera la percepción como simplemente el hecho representativo, interno o psicológico, como la conciencia en el sentido que hoy se la atribuye o el estado del espíritu que conoce lo que pasa en él Leibniz (apud MARTÍNEZ-FREIRE, 2010). La percepción es para Kant (1902) una especie de componente del término que acuña como apercepción, cuya definición es parecida a la de la percepción de los otros autores.

La apercepción se relaciona con tres clases de actividades mentales disponibles: las intuiciones, el conocimiento y los pensamientos (LAWLER, 2002). No es intuición porque esta es ciega y no pueden servir como fundamento para construir el entendimiento; no es conocimiento porque esta no es suficiente para el conocimiento, que requiere un acto de receptividad; es el pensamiento que precede y hace posible el conocimiento empírico, un pensamiento sin receptividad, totalmente espontaneo.

La significación de apercepción también se relaciona con todos los elementos ideales, que están latentes en el propósito de unificar el entendimiento y surgen con la percepción sensible para la objetivación del conocimiento empírico.

Con la apercepción se produce una de las habilidades antropológicas básicas: la de poder interpretar lo ya interpretado. Participan otros esquemas complementarios que sirven para la construcción de conocimiento, y proveen formas generales de información social: la memoria social conceptual, que corresponde a categorías abstractas de conocimiento y la memoria específica de eventos sociales (MONTERO, 2001). 


\subsection{Cultura de paz}

La paz, como derecho y deber, es un imperativo político, cívico, ético y pedagógico, la paz siempre es frágil y puede desaparecer, es un proceso continuo que se debe construir y vigilar constantemente y que requiere la participación de todos (UNESCO, 2011). Este debe ser un proceso de construcción permanente para la edificación de una cultura de paz.

Tres elementos sostienen la construcción de la paz: reconstrucción, resolución y reconciliación (GALTUNG, 1998). La reconstrucción solventa evidencias de la violencia directa; la resolución acuerda salidas a los saldos de la violencia estructural, y la reconciliación establece el diálogo y el entendimiento necesario para la convivencia y la paz (ROJAS VERA et al., 2006).

La cultura de paz no es un estado de armonía eterna, (KEMPF; GUTIÉRREZ VILLALOBOS, 2001) sino una clase de contrato social que permite a los miembros de la sociedad manejar (a nivel interno y externo) los conflictos dentro de un ambiente de cooperación.

Desde el punto de vista de la teoría sistémica, los conflictos son concebidos como sistemas complejos en los que elementos psicológicos, sociales y estructurales establecen dinámicas de relación, que se convierten en el motor que mantiene el sistema/conflicto en equilibrio y determina que se perpetúen (MÍNGUEZALCAIDE, 2017).

En esta perspectiva, se establece que la construcción de una cultura de paz se hace mediante la transformación del conflicto. "Requiere romper las dinámicas relacionales entre los elementos centrales del sistema/conflicto; a los que se les denomina atractores" (COLEMAN et al., 2007. p. 14). Dichos factores son estables, de gran consistencia, sostienen relaciones muy fuertes entre sí y con otros elementos del sistema/conflicto.

El sistema se sostiene con las dinámicas de relaciones entre los mecanismos psicológicos, sociales y estructurales de los individuos y los grupos (MINGUEZALCAIDE, 2017). Romper dichas dinámicas o atractores desequilibra el sistema, transforma el conflicto y facilita la construcción de paz (VALLACHER et al., 2010).

El proceso de esa construcción se debe hacer a partir del surgimiento de atractores de paz que generen nuevos equilibrios (NOVAK et al., 2012). Se requieren para una cultura de paz iniciativas con objetivos diversos, provenientes de múltiples 
grupos, que establezcan redes de acción a partir de procedimientos basados en la llamada democracia deliberativa con diálogos entre diferentes personas.

El rompimiento de atractores, percepciones, concepciones o ejercicios que históricamente se manejaban, es el elemento común de las propuestas en búsqueda del fortalecimiento o una cultura de paz. Para (KEMPF; GUTIÉRREZ VILLALOBOS, 2001) fortalecer la paz significa debilitar, o socavar las polaridades implícitas, antes de que el conflicto escale a violencia explícita.

Estos enunciados, para el caso de Colombia, permiten relacionar la política educativa con las políticas de la memoria del país, en un escenario en construcción, no exento de tensiones y discusiones, donde la presencia de los maestros es importante, pues son ellos, como sujetos pedagógicos, quienes materializan en el día a día los proyectos institucionales como la Ley de Víctimas y Restitución de Tierras y la Cátedra para la Paz en condiciones diversas (TORRES GÁRNEZ, 2016, p. 183).

Precisamente con el mismo enfoque, (GALTUNG, 1998) considera que para conseguir una cultura de paz hay que tejer las estructuras, pero no demasiado tirantes, no demasiado dominantes. Se requieren tres ingredientes básicos para construir una cultura de paz: no violencia, creatividad, y empatía. En la primera se responde a la violencia y la destrucción con algo constructivo, a la creatividad con la valoración de la vida y la promoción del otro y a la empatía con la capacidad de comprender al otro desde adentro.

En este tejido de paz, la pedagogía juega un papel de primera línea, pero como un ejercicio desde la experiencia, que no se encuentra planteado en algún libro, la escriben los y las maestras con sus prácticas cotidianas, ellos dan cuenta de que en una pedagogía para la paz se deben vincular estéticas de la sensibilidad, promover el cultivo de lo humano, de las emociones, de la empatía y la solidaridad, incentivar, seducir y lograr que los estudiantes no se sientan observadores o receptores del proceso, sino sujetos partícipes, compañeros en los procesos de aprendizaje (PEÑA CASTELLANOS, 2017).

Es el aula uno de los lugares desde donde se puede construir una cultura de paz, y son los maestros, actores activos de este proceso. La Educación tiene una gran responsabilidad en el cultivo de las emociones tendientes al bien común, se trata de "una educación que cultive la capacidad de apreciar el carácter humano, pleno e igual de cualquier otra persona" (NUSSBAUM, 2015, p. 16 apud PEÑA CASTELLANOS, 2017). En este sentido, una Educación para la paz necesariamente 
debe tener en cuenta las emociones, tanto la comprensión de aquellas que han estado presentes y legitiman el conflicto armado así como el cultivo de emociones proclives a la construcción de paz desde la escuela (USSBAUM, 2015, p. 57 apud PEÑA CASTELLANOS, 2017).

Ahora bien, la historia, el pasado, la memoria contribuyen también con este proceso, mediante la indagación crítica propuesta desde las acciones educativas precisas, detalladas, con ética y contenido, que metodológicamente proyectan según Bermúdez (2015 apud ARIAS GÓMEZ, 2016) el pensamiento crítico (p. 164).

El análisis de la historia reciente del país y los ejercicios de memoria en la escuela deben situarse en un enfoque integral y contextualizado que reconozca la presencia de las víctimas en los procesos pedagógicos. Esta concepción va de la mano de cambios en cuanto a la resignificación del concepto de poder como oportunidad para construir acuerdos, transformar realidades y reconstruir tejidos sociales irrumpidos por la guerra (TORRES GÁRNEZ, 2016, p. 182).

El rompimiento del que hablan los autores tiene que ver también con el lenguaje, en la intencionalidad, los conceptos e imaginarios que se construyen desde la transmisión y la recepción de procesos comunicativos (MONTERO, 2001). El entramado que se encuentra detrás de cada acción comunicativa opera en atención a motivaciones, normas, reglas y principios.

Los medios a través de los periodistas y la historia por intermedio de sus escritores, docentes y narradores, contribuyen al pesimismo y construcción de una visión unidireccional sobre la violencia/guerra y destrucción y se centran en una sola mirada, en las élites más que en el pueblo llano, se presenta a menudo a las élites como agresores y al pueblo como víctima en vez de una visión más equilibrada de ambos (GALTUNG, 1998).

Desde una visión sin equilibrio crítico se consolida un lenguaje dualista. La cultura de guerra dominada por un pensamiento dualista genera polaridades y se mueve en tres lógicas: manía jerarquizante, principio de la carencia, y transformación de lo diferente en inferioridad. En la organización jerárquica de la realidad, una de las polaridades domina a la otra, el mundo entonces se puede concebir como bidimensional: superior e inferior. La carencia se establece en relación con lo que se le asigna a la polaridad superior por lo general "nosotros", considerado como valioso y con entidad propia, lo "otro" que se clasifica como diferente, inferior, se le concibe como devaluado y sin identidad (KEMPF; GUTIÉRREZ VILLALOBOS, 2001). 
La transición de una cultura de guerra a una cultura de paz requiere alejarse del mundo bipolar creado por la modernidad; este alejamiento procura los elementos útiles para formular una lógica orientada a construir un pensamiento diversificado, sustentador de una cultura de paz, que se caracteriza por exaltar y respetar las diferencias de un mundo diverso, y la afirmación de la equidad.

El universo no puede pensarse dividido en dos campos, este ostenta una cantidad inabarcable de líneas de ruptura, de saltos, de abismos, en los cuales ya nadie sabe muy bien cómo orientarse: "el futuro es pluridimensional y los modelos explicativos de las generaciones anteriores no alcanzan como guía" (BECK apud SAINTOUT, 2007, p. 34).

Autores como Galtung (1998) y Bajaj (2008) consideran que, en contextos permeados por la violencia, además de trabajar en cuestiones como el desarrollo de habilidades interpersonales para la resolución de conflictos, o el reconocimiento y la valoración de la diversidad, la Educación para la paz, supone esencialmente, comprender y desaprender la guerra. Es por ello que coinciden en "la importancia de articular la enseñanza de la historia y la Educación para la paz con el desarrollo del pensamiento crítico que permita a los estudiantes desarrollar una comprensión más sofisticada de las causas, dinámicas y consecuencias de los conflictos sociales, reconocer empáticamente la experiencia de diversos actores y construir posiciones independientes sobre las opciones para abordar los conflictos" (PADILLA; BERMÚDEZ, 2016, p. 224).

En referencia concreta al papel de los docentes, profesores y maestros en la construcción de una cultura de paz, Torres Gárnez (2016) asegura que los conocimientos disciplinares no empoderan a los sujetos, no reparan ni construyen tejido social. Esto se logra pero con propuestas conscientes de formación de sujetos, con el desarrollo de habilidades cognitivas, reflexivas y afectivas que reconozcan la historia desde miradas críticas, imaginativas participativas y responsables.

\section{Método y materiales}

Esta investigación fue trabajada desde el paradigma cualitativo de tipo interpretativo, mediante el análisis de los resultados obtenidos por la triangulación de la información recibida. Se determinó un enfoque histórico hermenéutico, que en concepto de (CISTERNA CABRERA, 2005) es una forma de abordar, estudiar, analizar y construir conocimiento a partir de procesos de interpretación. Se asume la producción del conocimiento como un proceso subjetivo e intersubjetivo en el que se recopila información, se organiza y se le da sentido desde conceptualizaciones previas integradas a los hallazgos surgidos de la propia investigación. 
En primer lugar, se partió de considerar el valor de la Educación como un camino de transformación y de cambio social, para luego asumir el concepto de percepción, que plantea enfoques diversos según la disciplina, el momento histórico y las expectativas generadas, pero que se puede definir como un proceso cognitivo de la conciencia para el reconocimiento, interpretación y significación en la elaboración de juicios en torno a las sensaciones obtenidas del ambiente físico y social, complementada con otros procesos, como el aprendizaje, la memoria y la simbolización (VARGAS MELGAREJO, 1994).

En este contexto, se construyó una encuesta de tipo no estructurado, que se publicó en la plataforma Google Drive y se conectó con las páginas oficiales de la Universidad Cooperativa de Colombia, las redes sociales y correos institucionales. A través de ellos, mediante campañas de información, se motivó a la comunidad, incluidos los profesores que trabajaban en la institución, para que participaran libre y voluntariamente.

El cuestionario en la primera parte presentó interrogantes que caracterizaron a la población; la segunda registró seis preguntas, de las que tres respondieron a cuestionamientos relacionados con las percepciones. Dichas preguntas buscaron encontrar los imaginarios, las vivencias y las experiencias de una cultura de paz.

Dicho cuestionamiento hizo referencia a: ¿Qué episodios o hechos cree que limitan o restringen la construcción de la cultura de paz? Con esta pregunta los encuestados dieron cuenta de sus vivencias y experiencias relacionadas con los obstáculos para una cultura de paz.

En concordancia con (CISTERNA CABRERA, 2005), las categorías y subcategorías utilizadas en una investigación pueden establecerse desde antes o surgir en la medida que avanza el proyecto; precisamente este último postulado fue al que se acogió esta investigación.

Para la primera pregunta, se sintetizaron cinco categorías, que se desprendieron de la información vaciada en un documento Excel: 1. Intereses políticos y económicos, 2. Falta de Educación en valores, 3. Injusticia social, 4. Discriminación, 5. Priman los intereses particulares.

La segunda pregunta ¿Considera que los colombianos confían en el proceso de paz o en acuerdos frente a cualquier tipo de violencia? (Amplíe su respuesta), permitió que se interpretaran los imaginarios, con respecto a los acuerdos firmados. 
En este caso la pregunta generó dos opciones, para las respuestas con el no, se encontraron tres categorías: 1. No cambiarán, 2. No favorece al pueblo, 3. Tienen malos sentimientos. Para las respuestas con el sí, la única razón dada se sintetizó en: porque se quiere la paz.

La tercera pregunta $¿$ Piensa que la paz es una experiencia de vida y forma parte de la cotidianidad? Dicho cuestionamiento generó inferencias, que dieron muestra clara de la construcción, desde las influencias y caracterizaciones históricoculturales del contexto, de los encuestados.

Esta pregunta permitió dos alternativas que determinaron las dos categorías en las que se clasificó la información. En una, las respuestas positivas señalaron ambientes de paz en el contexto donde vivía, y en la otra, se incluyeron todas aquellas manifestaciones que formaban parte de la cotidianidad.

\subsection{Población}

En total se consideraron 602 docentes vinculados a la sede Bogotá y contratados bajo tres modalidades: de tiempo completo 313 profesores, 45 de medio tiempo y 244 de tiempo requerido o catedráticos como se les conoce.

Para este estudio de tipo cualitativo se consolidó la muestra con base en un cálculo estadístico, estratificado y proporcional de la población, teniendo en cuenta la cantidad y modalidad de contratos. La muestra de mínimos representativos se seleccionó mediante mapeo, con la intervención de criterios de inclusión y exclusión. Se aplicó a 250 personas vinculadas en calidad de administrativo o docente.

En el grupo de administrativos y docentes, fueron estos quienes en mayor cantidad se hicieron presentes; 158 profesores participaron, de los cuales 129 contestaron todas las preguntas acerca de percepciones.

Para la sistematización se utilizaron tres procedimientos. Inicialmente se recolectó la información a través de la plataforma virtual de Google Drive; la comunidad académica intervino de forma voluntaria, con unos mínimos de participación, por cada uno de los programas, departamentos y actividades, lo que garantizó la representación equilibrada de todos los actores.

En segunda instancia, se hizo un vaciado de la información recogida, en plantillas de Excel, que facilitaron la visualización y la clasificación por categorías. Dicha información se organizó en tablas dispuestas con las respuestas de cada una de las preguntas. 
El proceso siguiente consistió pasar la información, de las plantillas de Excel al software Atlas.ti, utilizado como herramienta de análisis de discurso; se graficaron las redes surgidas desde cada una de las variables y además se pudo hacer cruce comparativo desde diversas intersecciones de la información.

\section{Resultados}

En la encuesta total aplicada participaron 158 docentes, y las preguntas referentes a las percepciones fueron contestadas por 129 profesores. Para sistematizar las respuestas, se organizaron cinco grupos por rangos de edad: más de 60 años, de 51 a 60 años, de 41 a 50 años, de 31 a 40 años y de 18 a 30 años.

En cada uno de los grupos de edad se hizo análisis de las tres preguntas correspondientes a percepciones. Se consideró la edad como la variable organizadora teniendo en cuenta varios elementos: los periodos de violencia vividos por cada una de las edades fue diferente, el lenguaje utilizado por los docentes era distinto según el rango de edad. Las didácticas y pedagogías diferían entre una y otra edad; la construcción histórica de vida tuvo diversas miradas según la experiencia y los años vividos.

En el primer grupo de profesores que tenían más de 60 años, contestaron la encuesta de percepciones un total de nueve docentes: dos mujeres y ocho hombres todos con estudios de posgrado.

En relación a las preguntas sobre percepciones, cuatro personas ubicadas en este rango de edad consideraron que los hechos que limitan la cultura de paz eran la falta de Educación y ausencia de valores; dos profesores indicaron que la discriminación e intolerancia fueron las causantes de esta situación; los otros tres encuestados creían que los intereses particulares, la injusticia y los intereses políticos eran los obstáculos para implementar una cultura de paz.

En cuanto a la segunda pregunta, acerca de la confianza en el proceso de paz, dos personas contestaron que sí había confianza, debido a los esfuerzos para implementarla y porque los colombianos la necesitan. Las restantes siete contestaron que no, ya que el proceso no era muy claro y todavía tenía muchos enemigos.

En relación con la tercera pregunta; ocho profesores manifestaron que efectivamente la paz era una experiencia cotidiana, ya que durante los últimos años "es de lo único que se ha oído hablar"; un docente afirmó que no forma parte de la cotidianidad porque simplemente es un anhelo, un deseo buscado permanentemente. 
En el segundo grupo, es decir del rango 51 a 60 años, contestaron la encuesta 36 profesores; 24 mujeres y 12 hombres, quienes a nivel educativo se clasificaban como: un docente con título de doctor, 18 con título de magister y los restantes con especialización.

Para este segmento de la población, los postulados que restringían la construcción de una cultura de paz se relacionaban con la falta de Educación y la ausencia de formación en valores; en ese ítem se contaron 19 respuestas. El segundo elemento considerado por 11 personas fue el de los intereses particulares, políticos y económicos y respecto a las diferencias e injusticia sociales, cuatro personas la dieron como razón; finalmente dos profesores dijeron que los medios de comunicación son los principales obstáculos para la construcción de una cultura de paz.

Respecto a si los colombianos confiaban en los procesos de paz, se presentó casi un equilibrio en las respuestas. 15 docentes consideraron que los colombianos eran positivos respecto a esta propuesta porque "[...] es hora que todos nos volvamos a mirar a los ojos sin rencor"; 14 docentes negaron esta opción, "han pasado décadas de engaño y promesas". Los siete restantes no manifestaron una posición clara.

Se obtuvo una respuesta, en su mayoría positiva, respecto a la pregunta de si la paz era una experiencia cotidiana de la vida; 28 docentes contestaron que, por trabajar en un centro de Educación Superior, esta actitud debía ser casi una obligación.

El siguiente grupo de docentes encuestados, correspondió al segmento de 41 a 50 años. Participaron 38 docentes, 12 de género femenino y 26 de masculino. A nivel educativo dos eran profesionales, nueve especialistas, 25 magister y dos doctores.

El mayor número de encuestados en este segmento, 14 en total, consideraron los problemas políticos, el poder, la corrupción, como los obstáculos para construir una cultura de paz. En segundo lugar, ubicaron el factor de discriminación, intolerancia, creencias, deseos de venganza. Otro elemento que tuvieron en cuenta varios participantes fue la ausencia de valores, poca Educación, y mínima información.

En este grupo, 21 encuestados consideraron que no existía confianza para apoyar el proceso de paz, pensaban que "los actores principales del conflicto actuaron a espaldas de la democracia y le mintieron al pueblo". De forma positiva se manifestaron 17 profesores quienes en términos generales consideraron que "después de 60 años de guerra esta, era una oportunidad". Solo cuatro personas 
manifestaron su oposición al sí, como expresión de que la paz debe ser una experiencia y un proceso de la cotidianidad.

El cuarto grupo de docentes entre 31 y 40 años estuvo conformado por 38 profesores mitad hombres y mitad mujeres. En relación con su formación académica, cinco terminaron un doctorado, 23 una maestría, ocho una especialización y dos eran profesionales.

En total 20 encuestados consideraron que el obstáculo que limitaba la construcción de una cultura de paz era la corrupción, la mala política, el poder; otras 14 personas relacionaron la discriminación y falta de tolerancia.

En relación con la confianza que los colombianos tenían en el proceso, apenas cinco contestaron que sí existe la confianza; el resto consideró que esta situación no favorecía al pueblo colombiano.

Una circunstancia parecida se presentó con la respuesta tres. En este caso las variables se invirtieron. La mayoría consideró que la paz era una experiencia cotidiana, y por el no, votaron apenas cinco personas.

Finalmente, en el grupo más joven, integrado por docentes entre los 18 y 30 años, participaron 32 docentes, 11 hombres, 20 mujeres y uno en el ítem de otros. Contestaron la encuesta 25 profesores. En este grupo, el nivel de formación en su mayoría (10) era de técnicos y tecnólogos; cuatro dijeron haber terminado la secundaria, seis eran profesionales, tres tenían especialización y cuatro se titularon de maestría.

Acerca de los obstáculos para la construcción de una cultura de paz: 11 profesores consideraron que la discriminación, la venganza, el rencor, la intolerancia eran las principales causas. Seis profesores manifestaron que la injusticia social, la falta de oportunidades y la inequidad eran los principales impedimentos.

De los 25 profesores que contestaron la encuesta, 15 manifestaron que los colombianos no confiaban en los procesos de paz. Seis profesores dijeron que personalmente confiaban, pero creían que el resto de los ciudadanos no lo hacían. Cuatro personas respondieron afirmativamente, sin restricciones.

En la tercera pregunta, la respuesta que tuvo mayor acogida fue la de que sí es una experiencia que beneficia y mejora la calidad de vida de todos y es el propósito de búsqueda común. Cuatro personas contestaron no, sin ningún tipo de explicación. 
Tabla 1 - Percepciones en docentes de universitarios

\begin{tabular}{lccccc}
\hline Preguntas y Categorías & $\begin{array}{c}\mathbf{1 8 - 3 0} \\
\text { años }\end{array}$ & $\begin{array}{c}\mathbf{3 1 - 4 0} \\
\text { años }\end{array}$ & $\begin{array}{c}\mathbf{4 - 5 0} \\
\text { años }\end{array}$ & $\begin{array}{c}\mathbf{5 1 - 6 0} \\
\text { años }\end{array}$ & $\begin{array}{c}\text { Mas } \mathbf{6 0} \\
\text { años }\end{array}$ \\
\hline ¿Qué episodios o hechos cree que limitan o restringen la construcción de la cultura de paz? \\
\hline Intereses políticos y económicos & 6 & - & 14 & 11 & 1 \\
falta de Educación en valores & 2 & 4 & 12 & 19 & 4 \\
Injusticia social & - & - & - & 4 & 1 \\
Discriminación & 11 & 14 & 12 & - & 2 \\
Priman los intereses particulares & 6 & 20 & - & 2 & 1 \\
\hline Total & 25 & 38 & 38 & 36 & 9 \\
\hline
\end{tabular}

¿Considera que los colombianos confían en el proceso de paz o en acuerdos frente a cualquier tipo de violencia?

\begin{tabular}{lcccccc}
\hline No- No cambiaran/ no posición clara & - & - & - & 7 & - \\
No- No favorecen al pueblo & 15 & 33 & 21 & 14 & 7 \\
No- Tienen malos sentimientos & - & - & - & - & - \\
Si-porque se quiere la paz & 10 & 5 & 17 & 15 & 2 \\
\hline Total & 25 & 38 & 38 & 36 & 9 \\
\hline ¿Piensa que la paz es una experiencia de vida y forma parte de la cotidianidad? & \\
\hline Si. Ambientes de paz donde se vive & 21 & 33 & 34 & 28 & 8 \\
No. Debería ser parte, pero no ocurre & 4 & 5 & 4 & 8 & 1 \\
\hline Total & 25 & 39 & 38 & 36 & 9 \\
\hline
\end{tabular}

Fuente: Elaboración propia (2019)

\section{Análisis y discusión}

Realmente en la respuesta a la pregunta acerca de cuáles son las percepciones que subyacen en la comunidad académica universitaria respecto a la cultura de paz y en referencia a los docentes, es clara la diferencia respecto al factor edad.

Los docentes con más de 60 años y los que se ubicaron en el rango entre 51 a 60 años manifestaron que la falta de Educación y la carencia de valores son las principales razones que limitan la construcción de una cultura de paz. Refleja esto la construcción de imaginarios desarrollados desde sus propios valores y experiencia. Esta apreciación no tiene casi validez para los más jóvenes porque como dice (VARGAS MELGAREJO, 1994), las percepciones al igual que las representaciones son esquemas de interpretación, valoración y clasificación del conocimiento del mundo 
Quienes se ubicaron en los rangos de entre 41 y 50 y entre 31 y 40 años, consideraron que los problemas políticos causados por el poder y la corrupción eran el principal obstáculo para construir cultura de paz. Los docentes más jóvenes cuya edad oscilaba entre 18 y 30 años pensaban en la discriminación, y la intolerancia, como las principales razones que limitan la cultura de paz.

Efectivamente, elementos como la corrupción, discriminación, la intolerancia, son temas que se acercan más a los intereses de los más jóvenes. Ellos se educan y trabajan en contextos donde este tipo de problemáticas están presentes.

Las construcciones de crianza, las precepciones y concepciones, así como los imaginarios, son los elementos que constituyen los "atractores" del sistema/ conflicto y que se requiere romper para construir nuevas dinámicas relacionales de una cultura de paz. Solo será posible desde la academia si la comunidad, empezando por los docentes, se concientiza de la "carga" que cada uno lleva.

Un análisis del lenguaje y específicamente de las palabras empleadas en relación con los imaginarios para la construcción de una cultura de paz, encontradas en la encuesta realizada en el marco de esta investigación, buscó establecer cómo desde el lenguaje se podría generar el rompimiento para el cambio de cultura que, desde la intencionalidad, muestre que los conceptos e imaginarios se construyen para la transmisión y la recepción de procesos comunicativos (MONTERO, 2001).

De las 9.905 palabras que en total se escribieron en la encuesta, las relacionadas directamente con la temática en cuestión y las que en mayor proporción se repitieron fueron: paz con 543 repeticiones, proceso 185, vida 170 y cultura 160 . En un segundo nivel aparecieron palabras relacionadas con el conflicto como violencia 29 repeticiones, guerra 26 y conflicto en 21 ocasiones.

Es perfectamente debatible el hecho de que la mayoría de los docentes manifestó que existen muchos obstáculos y aunque no consideran la paz como viable se reflejó una gran esperanza, en su lenguaje. Las expresiones relacionadas con la violencia, con el conflicto, con la guerra, se quisieron evitar. Niklas Luhmann manifestaba que en el lenguaje no solo se debe tener en cuenta de "qué se trata el caso" sino también de "qué es lo que se esconde detrás". Las palabras intereses, intolerancia y corrupción aparecieron apenas 12 o 15 veces cada una.

De forma general, los docentes perciben que los colombianos no confían en el proceso de paz porque el proceso no es muy claro, tiene muchos enemigos, y se actúa a espaldas de la democracia, causado por el poco acceso a la Educación, la 
carencia de valores, que genera abuso de poder, corrupción y exclusión. Como dice Bourdieu (2007) el habitus se construye desde una concepción del poder y las percepciones y acciones del mundo se marcan desde la dotación de determinados habitus que el sujeto o actor social tengan para sí. Sin embargo, los docentes en esta investigación concluyeron que su actividad los involucra, con hacer de la paz un ejercicio cotidiano. 


\section{Percepções de professores universitários para a construção de uma cultura de paz}

\section{Resumo}

A investigação apresentada busca determinar as percepções subjacentes à comunidade do professor universitário sobre a cultura da paz. Em um processo de transformação da cultura da violência, com a responsabilidade de contribuir para a transmissão dos elementos para uma construção que gere mudança social. A pesquisa foi trabalhada a partir do paradigma qualitativo, do tipo interpretativo e da análise dos resultados obtidos pela triangulação de uma pesquisa não estruturada, aplicada aos professores, que participaram de forma livre e voluntária. As pessoas foram questionadas sobre os episódios que limitam ou restringem a construção de uma cultura de paz, a percepção que têm sobre os acordos, para saber se confiam ou não neles e, finalmente, se essa cultura de paz está considerando uma experiência de vida que faz parte do cotidiano dos envolvidos. Os professores concordam, principalmente, que os colombianos não confiam no processo de paz porque não é bem explicado e tem muitos inimigos. Eles disseram que os principais atores da guerra fizeram coisas pelas costas da democracia e até mentiram para os cidadãos. A idade foi considerada como variável organizadora, levando em consideração vários elementos. Em primeiro lugar, os períodos de violência vividos por cada grupo de idades foram diferentes, a linguagem utilizada pelos professores é diferente de acordo com a faixa etária. Em segundo lugar, as didáticas e as pedagogias diferem entre uma e outra e a construção histórica da vida tem visões diferentes de acordo com a experiência e os anos vividos.

Palavras-chave: Percepções. Cultura de Paz. Mudança Social. Paz. Ensino. 


\section{University teacher perceptions for a peace culture construction}

\section{Abstract}

The investigation seeks to determine the perceptions that underlie a community of university professors about the culture of peace. The scenario is a process of transformation of a violence culture with the responsibility of contributing to the transmission of elements that generates social change. The research follows a qualitative paradigm of interpretative type and the analysis of the results is obtained by the triangulation of an unstructured survey applied to the teachers, who participated freely and voluntarily. People were asked about the episodes that limit or restrict the construction of a culture of peace, the perception they have about the agreements, in order to know if they trust or not in them, and finally, if the peace is considered a life experience that is a part of daily life. The professors mostly agree that Colombians do not trust the peace process because it is not well explained and it has many enemies. They said that the main actors in the war do things that do not support democracy and that they even lie to the citizens. The age was considered as the organizing variable taking into account several elements. In the first place, the periods of violence lived by each of the group of ages were different, the language used by the teachers is different according to the age range. In the second place, the didactics and pedagogies differ between one age and another, and the historical construction of life has different views according to the experience and the years lived.

Keywords: Perceptions. Culture of peace. Social Change. Peace. Teaching. 


\section{Referencias}

ARIAS GÓMEZ, D. H. La memoria y la enseñanza de la violencia desde estrategias audiovisuales. Revista Colombiana de Educación, Bogotá, n. 71, p. 253-278, 2016. Disponible en: http://www.scielo.org.co/pdf/rcde/n71/n71a10.pdf. Acceso en: 27 jul. 2019.

BAJAJ, M. Critical peace education. In: BAJAJ, M. (ED.). Encyclopedia of peace education. Greenwich: Age, 2008. p. 135-146.

BOURDIEU, P. El sentido práctico. Buenos Aires: Siglo XXI, 2007.

BOURDIEU, P. La distinción: criterios y bases sociales del gusto. Madrid: Taurus. 1988.

BOQUÉ TORREMORELL, M. C., et al. La cultura de paz en la Educación para la ciudadanía y los derechos humanos en los libros de texto de Educación primaria. Perfiles Educativos, México, DF, v. 36, n. 146, p. 80-97, 2014. Disponible en: http://www.redalyc.org/articulo.oa?id=13232069006. Acceso en: 20 ene. 2018.

BRUNER, J. Actos de significado más allá de la revolución. Madrid: Alianza, 1990.

CISTERNA CABRERA, F. Categorización y triangulación como procesos de validación del conocimiento en investigación cualitativa. Theoría. Chillán, v. 4, n. 1, p. 61-71, 2005. Disponible en: http://www.redalyc.org/articulo. oa?id=29900107. Acceso en: 27 feb. 2017.

COLEMAN, P.T. et al. Intractable conflict as an attractor: a dynamical systems approach to conflict escalation and intractability. American Behavioral Scientist, Thousand Oaks, v. 50, n. 11. p. 1454-1475, Jul. 2007. https://doi.org/10.1177/0002764207302463

GALTUNG, J. Tras la violencia 3R: reconstrucción, reconciliación, resolución. Bilbao: Gernika Gogoratuz, 1998. (Colección Red Gernika).

KANT, I. Kant's gesammelte Schrift en pequeñas percepciones e ilustración en leibniz y Kant. Berlin: Königlich Preußischen Akademie der Wissenschaften und ihren Nachfolgern; 1902.

KEMPF, W.; GUTIÉRREZ VILLALOBOS, S. Los medios y la cultura de paz. Berlin: Regener; 2001. 
LAWLER, D. Kant, autoconciencia y actualidad del principio de apercepción en la literatura analítica reciente. Azafea: Revista de Filosofia, Salamanca, v. 4, p. 211-239, 2002. httpa://doi.org/10.14201/3737

MARTÍNEZ-FREIRE, P. F. La naturaleza representacional de la mente según Leibniz. In: SÁNCHEZ RODRÍGUEZ, M.; RODERO, S. (eds.). Leibniz en la filosofía y la ciencia modernas. Granada: Comares, 2010. p. 247-263.

MÍNGUEZ-ALCAIDE, X. Un proceso de diálogo multiactor para la paz en el País Vasco. Convergencia, Toluca, v. 24, n. 73, p. 37-60, ene./abr.2017. Disponible en: http://www.scielo.org.mx/scielo.php?script=sci_arttext\&pid =S1405-14352017000100037. Acceso en: 15 jul 2017.

MONTERO, L. Prefacio. In: KEMPF, W.; GUTIÉRREZ VILLALOBOS, S. Los medios y la cultura de paz. Berlin: Regener; 2001. p. 7-12.

NOWAK, A. et al. Sustainable peace: a dynamical systems perspective. In: COLEMAN, P. T.; DEUTSCH, M. (eds.). Psychological components of sustainable peace. [S. l.]: Springer Science Business Media, 2012. p. 265-281. (Peace psychology book series).

PADILLA, A.; BERMÚDEZ, A. Normalizar el conflicto y desnormalizar la violencia: retos y posibilidades de la enseñanza crítica de la historia del conflicto armado colombiano. Revista Colombiana de Educación, Bogotá, n. 71, p. 219-251, jul./dic. 2016. Disponible en: http://www.scielo.org.co/scielo.php?script=sci_arttext \&pid=S0120-39162016000200009. Acceso en: 27 jul. 2019.

PEÑA CASTELLANOS, M. Geopolítica de las emociones: Narrativas de maestras sobre conflicto armado y construcción de paz en la escuela. Revista Ciudad Paz-ando, Bogotá, v. 10, n. 2, p. 54-61, jul.-dic. 2017. https://doi.org/10.14483/2422278X.12798

ROJAS VERA, L. R., et al. Comunicación, conflictos y cultura de paz: percepción en grupos de estudiantes universitarios. Reflexión Política, Bucaramanga, v. 8, n. 15, p. 52-63, jun. 2006.

SAINTOUT, F. J. Jóvenes e incertidumbres: percepciones de un tiempo de cambios: familia, escuela, trabajo y política. Tesis (Doctorado en Ciencias Sociales) - Facultad Latinoamericana de Ciencias Sociales, Buenos Aires, 2007.

TORRES GÁRNEZ, L. Historia reciente en la escuela colombiana: acercamiento a las nociones de memoria, historia y conflicto. Revista Colombiana de Educación, Bogotá, n. 71, p. 165-185, 2016. Disponible en: http://www.scielo.org.co/pdf/rcde/n71/n71a07.pdf. Acceso en: 17 ene. 2018. 
UNESCO. Informe final. In: REUNIÓN DE CONSULTA SOBRE EL PROGRAMA DE CULTURA DE PAZ, 1., Paris, 1994. Disponible en: http://unesdoc.unesco.org/images/0010/001001/100167sb.pdf. Acceso en: 17 ene. 2018.

UNESCO. Unesco's transdisciplinary project "Towards a Culture of Peace”. Paris: Unesco, 1998. Disponible en: http://unesdoc.unesco.org/images/0011/001177/117753eo.pdf. Acceso en: 17 ene. 2018.

UNESCO. Cómo contribuye la UNESCO a edificar una cultura de paz y al desarrollo sostenible. In: UNSECO GENERAL CONFERENCE, 36., 2011, Paris. Documento de información[...] Disponible en: http://unesdoc.unesco. org/images/0021/002138/213800s.pdf. Acceso en: 17 ene. 2018.

VALLACHER, R. R. et al. Rethinking intractable conflict: the perspective of dynamical systems. American Psychologist, Washington, DC, v. 65, n. 4, p. 262-278, may-june 2010. https://doi.org/10.1037/a0019290

VARGAS MELGAREJO, L. M. Sobre el concepto de percepción. Alteridades, México, DF, v. 4, n. 8, p. 47-53, 1994. Disponible en: http://www.redalyc.org/pdf/747/74711353004.pdf. Acceso en: 17 ene. 2018.

VYGOTSKY, L. S. El desarrollo de los procesos sicológicos superiores. Barcelona: Crítica, 1978. 


\section{Información de los autores}

María Elsa Unriza Puin: Doctoranda por el Programa de Postgrado conocimiento y Cultura de America Latina pelo Instituto Pensamiento y Cultura en América Latina-Mexico. Docente investigadora de la Universidad Cooperativa de Colombia, sede Bogotá. Contacto: maria.unriza@ucc.edu.co

iD https://orcid.org/0000-0001-6477-1849

Isabel Hernández Arteaga: Posdoctorado en investigación cualitativa, Doctora en Ciencias de la Educación. Docente Escuela de Posgrados en Educación de la Universidad Cooperativa de Colombia sede Bogotá. Investigadora del Centro de Investigación en Docencia Universitaria. Contacto: isabel.hernandez@ucc.edu-co

iD https://orcid.org/0000-0002-6299-0236

Fredys Alberto Simanca Herrera: Magister en Informática Aplicada a la Educación. Docente Investigador programa de Ingeniería de Sistemas de la Universidad Cooperativa de Colombia sede Bogotá. Contacto: fredys.simanca@campusucc.edu.co

iD https://orcid.org/0000-0002-3548-0775 